\title{
DISCUSSION V.7 ON IRREGULAR GALAXIES
}

\section{KINMAN: HI OBSERVATIONS OF DWARF GALAXIES}

Few dwarf galaxies $\left(M_{\mathrm{pg}}>-15\right)$ are known outside our Loca1 Group. A1though Fisher and Tu11y (1974, A.A. 44, 151) detected HI by the $21-\mathrm{cm}$ line in 179 out of the 243 objects in the DDO catalog of dwarf ga1axies, on1y 8 percent of those detected have $\mathrm{M}_{\mathrm{pg}}>-15$, and on1y 15 percent have $\mathrm{M}_{\mathrm{pg}}>-16$. The Arecibo radio telescope was therefore used by E.K. Conklin and myself at $21 \mathrm{~cm}$ in the velocity range -500 to $1500 \mathrm{~km} / \mathrm{s}$ to observe dwarf galaxy candidates to a hydrogen flux 1imit of $\sim 4 \times 10^{4} \mathrm{M}_{\odot} \mathrm{Mpc}^{-2}$ (velocity resolution $4 \mathrm{~km} / \mathrm{s}$ ); photoelectric magnitudes and colors were obtained at KPNO. Among candidates chosen for low surface brightness, HI was found in three out of seven objects taken from Karachentsava's (1968, Comm. Byurakan Obs. 39, 61) catalog of Sculptor systems. These are dwarf irregular galaxies with $\mathrm{M}_{\mathrm{pg}}=$ $-12.8,-14.4$, and $-15.5\left(H_{O}=50 \mathrm{~km} \mathrm{sec}^{-1} \mathrm{Mpc}^{-1}\right)$, which suggests that Karachentsava's catalog (like the DDO catalog) consists primarily of late-type systems. HI was also found in one DDO galaxy (No. 72, $\mathrm{M}_{\mathrm{pg}}=$ -15.6) out of four searched, in which none had been detected by Fisher and Tully. Among compact, blue emission-line galaxies, neutral hydrogen was found in seven out of eight low-velocity galaxies discovered by Rubin et a1. (1967, A.J. 72, 59) in the direction of the Virgo cluster. One of these (RMB 132) is a satellite of M87. A provisional comparison of the radio and optical properties of these compact galaxies with some low-velocity Markarian galaxies supports the idea that these RMB objects are members of the Virgo cluster with $M_{p g}$ in the range -14.1 to -16.6 . Spectroscopic observations are in progress in collaboration with K. Davidson and R.M. Humphreys.

TULLY: GAS DISTRIBUTION, MOTIONS AND DYNAMICS FOR SOME DWARF IRREGULAR GALAXIES

The magellanic irregular galaxies DDO $125\left(\mathrm{M}_{\mathrm{pg}}=-15 \mathrm{~m}_{9}\right)$ and Ho I $\left(M_{\mathrm{pg}}=-14 \mathrm{~m}_{4}\right)$ have been observed with the Westerbork Synthesis Radio Telescope in the $21-\mathrm{cm}$ HI line. These systems are intrinsically the faintest such objects for which we now have high-resolution kinematic information. Both galaxies are found to have well-ordered velocity fields which can be described as solid-body rotation over most of the disk, with a hint of a velocity turnover toward the extremities. For both, mass models have been fitted, based on gaussian density distributions characterized by a central density and scale lengths in and perpendicular to the disk. In both galaxies the random gas motions are comparable to the rotation velocities and provide a significant pressure term in the equations of motion. The masses derived are of order $5 \times 10^{8} \mathrm{M} \odot$, and the $\mathrm{M} / \mathrm{L}$ ratios are of order 3 in both systems. These values should be considered as lower limits since considerable mass might be located at radii exceeding the scale length. Similar gaussian models have been fitted to the previously observed irregulars: the Sma11 Magellanic Cloud, NGC 3109 and NGC 6822. The 1arge-scale features of all five galaxies are compared. A more extensive discussion is in press (1978, A.A.). 
HEIDMANN: MASS OF CLUMPS IN THE IRREGULAR GALAXY MARKARIAN 296

In a previous work we drew attention to a new class of irregular galaxies which are larger, brighter and with larger internal motions than classical irregulars, have UV emission and are characterized by a clumpy structure made up of half a dozen of clumps scattered in a common envelope. On1y six cases are known so they appear to be rare. It was suggested that these giant clumpy irregular galaxies could still be in a transient state of evolution with large cells where the rate of star formation is high (Casini and Heidmann 1976, A.A. 47, 371).

C. Casini and I obtained a high dispersion $\mathrm{H \alpha}$ spectrum and the 21$\mathrm{cm}$ line profile of one of them, Markarian 296. The spectrum shows a linear velocity gradient of $120 \mathrm{~km} / \mathrm{s}$ along the line of $8 \mathrm{clumps}$, which is $5 \mathrm{kpc}$ long. If stable, and with a reasonable inclination, the system of clumps has a total mass $1.2 \times 10^{9} \mathrm{M}_{\odot}$. This gives for each clump and its neighbourhood a mean mass $1.5 \times 10^{8} \mathrm{M}_{\odot}$.

The HI mass of the galaxy is three times larger, $3.4 \times 10^{9} \mathrm{M}_{\odot}$. Thus neutral hydrogen is probably scattered in a larger volume and it would be interesting to map it with the Westerbork radiotelescope.

A more complete study of this galaxy with low dispersion spectra obtained by $M$. Tarenghi is in preparation in collaboration with him.

BURBIDGE: What is the $\mathrm{M} / \mathrm{L}$ ratio?

HEIDMANN: For the system of clumps, the M/L ratio is very small. If one uses what we call the indicative total mass for the total mass of the galaxy, as evaluated from the width of the $21-\mathrm{cm}$ line and the optical dimensions, the $\mathrm{M} / \mathrm{L}$ ratio is 10 . But of course it would be nicer to evaluate the total mass from a kinematical mapping of the neutral hydrogen with the Westerbork telescope.

LANDECKER: HI OBSERVATIONS OF THE IRREGULAR GALAXY NGC 4214

Aperture synthesis observations have been made of neutral hydrogen and continuum emission from the type I irregular galaxy NGC 4214 by P.E. Dewdney and myself. Angular resolution is $2 \times 4$ arcmin and velocity resolution $5 \mathrm{~km} / \mathrm{s}$.

The hydrogen distribution shows a marked concentration to the center of the galaxy, coinciding with the bulk of the optical emission and a weak continuum source. There is clear evidence for rotation, and a well defined axis and center of rotation have been found. The profiles obtained suggest a thick spheroidal distribution as an appropriate model. However a Brandt rotation curve is asymmetrical and cannot easily be fitted to the data. Only a lower limit to the mass has been obtained.

Optical parameters

Distance

Holmberg Size

Derived parameters are

Neutra1 Hydrogen Extent

Systemic Velocity

Rotation Axis

Inclination to Line of Sight
6. $3 \mathrm{Mpc}$
$10.6 \times 10.6$ arcmin
$18 \times 13$ arcmin
$302 \pm 5 \mathrm{~km} / \mathrm{s}$
$15^{\circ} \mathrm{W}$ of North $\pm 5^{\circ}$
$39^{\circ} \pm 5^{\circ}$ 
Continuum F1ux

Neutra1 Hydrogen Mass

Tota1 Mass Lower Limit
$50 \pm 10 \mathrm{mJy}$

$3 \times 10^{9}$ solar masses

$5 \times 10^{9}$ solar masses

\section{SEAQUIST: RADIO RECOMBINATION LINES IN M82}

M.B. Bell and I have detected the $\mathrm{H} 102 \alpha(6.1 \mathrm{GHz})$ and $\mathrm{H} 85 \alpha(10.5$ $\mathrm{GHz})$ lines in M82 with the Algonquin 46-meter telescope. These results confirm the existence of observable recombination 1 ines in M82, in agreement with our earlier work (Be11 and Seaquist 1977, A.A. 56, L461) at $\mathrm{H} 1 \mathrm{O} 2 \alpha$ and the work of Shaver, Churchwe11, and Walmsley (1977, preprint) at $\mathrm{H} 166 \alpha(1.4 \mathrm{GHz})$ and $\mathrm{H} 110 \alpha(4.9 \mathrm{GHz})$. Figure 1 shows our results at both $900 \mathrm{kHz}$ and $300 \mathrm{kHz}$ resolution.
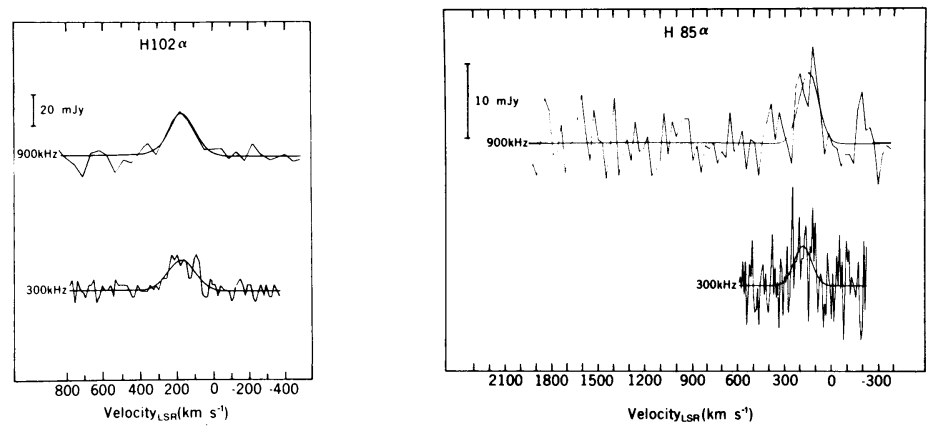

When the observed peak intensities $(24 \pm 4 \mathrm{mJy}$ at $\mathrm{H} 102 \alpha ; 7.5 \pm 2 \mathrm{mJy}$ at $H 85 \alpha$ ) are combined with the existing data on M82 at $H 166 \alpha$ and $H 110 \alpha$ made with a single paraboloid, the peak intensities show a decrease with increasing frequency. This decrease is inconsistent with an origin for the lines by spontaneous emission, but is consistent with stimulated emission involving ionized gas in front of the nonthermal radio source in the nucleus of $\mathrm{M} 82$, as originally suggested by Shaver, Churchwe11 and Rots (1977, A.A. 55, 435).

The mean velocity width $(160 \pm 40 \mathrm{~km} / \mathrm{s})$, and centroid ( $\mathrm{V}_{\mathrm{LSR}}=177 \pm 20$ $\mathrm{km} / \mathrm{s}$ ) from Figure 1 are consistent with the known properties of ionized gas in the nucleus. We are currently developing a simple model to account for the recombination line data.

VAN DER HULST: The mean velocity of the emission lines (H110 $\alpha$, H102 $\alpha$, $\mathrm{H} 85 \alpha$ ) observed with a single dish is about $192 \mathrm{~km} / \mathrm{s}$. You suggest that the emission is arising from a small central region in M82 (i.e. the region outlined by the radio continuum map of Kronberg et a1.). The

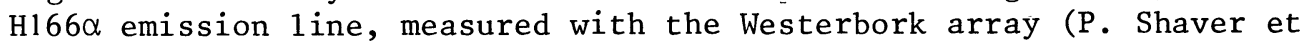
a1. 1976) comes from about the same region. Yet the mean velocity of the $H 166 \alpha$ line is about $240 \mathrm{~km} / \mathrm{s}$. Do you have an explanation for this difference?

SEAQUIST: Within the nuclear region there is a velocity spread of at least $200 \mathrm{~km} / \mathrm{s}$, and it is possible that different regions contribute at different frequencies. On the other hand, the difference may not be 
real; because of the low signal-to-noise ratio of the profiles the velocities may not be determined as well as we think.

GIOVANELLI: You showed us several profiles in two different resolutions. Were they independent measurements, confirming each other, or the same signal observed with different bandwidths?

SEAQUIST: No, they are not dependent data. We used one front-end connected to two spectrometers in parallel.

GIOVANELLI: You quoted errors of 10 or $20 \mathrm{~km} / \mathrm{s}$ for the 1 ines' central velocity, and the widths are as large as $300 \mathrm{~km} / \mathrm{s}$. Since several 1 ines were on $1 y$ a few channels wide, how can you determine central velocities with such accuracy?

SEAQUIST: The error quoted is half the velocity resolution. I don't think this is unreasonable.

COMTE: PRELIMINARY RESULTS FROM AN H $\alpha-[N I I]$ LINE SPECTROSCOPIC STUDY OF $\mathrm{M} 82$

Spectra of the system of emissive filaments in the galaxy M82 have been taken with the image-tube nebular spectrograph attached to the Cassegrain focus of the 193-cm telescope in Haute Provence. The preliminary results of this survey are:

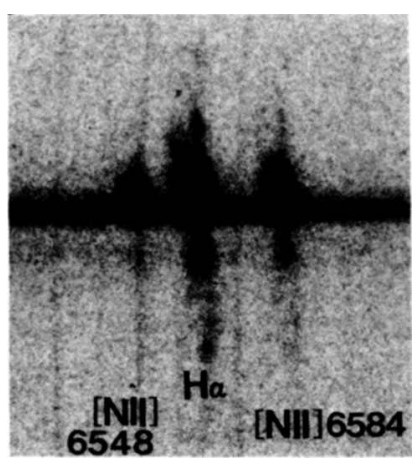

(1) Up to \pm 2 ' from the adopted "nucleus", the $\mathrm{H} \alpha /[\mathrm{NII}]$ line intensity ratio is not significantly different from Peimbert and Spinrad's value.

(2) The velocity field of the filaments up to $\pm 3^{\prime}$ is very complex but the main outline is consistent with previous measurements by other authors, and recent $21-\mathrm{cm}$ line data, i.e. blueshift with respect to the systemic velocity on the southern side and redshift on the northern side.

(3) Detailed analysis of the $\mathrm{H} \alpha$ and [NII] line profiles in the filaments show several ( 3 to 4 ) velocity components within a line, becoming more and more resolved with increasing distance from the center. Each component shows a halfmaximum linewidth superior to the instrumental response $(75 \mathrm{~km} / \mathrm{s})$; the internal velocity dispersion of components varies, but does not exceed $150 \mathrm{~km} / \mathrm{s}$.

OORT: What are the velocity differences between the components?

COMTE: About $300 \mathrm{~km} / \mathrm{s}$.

SCARROTT: A DUST SCATTERING MODEL OF M82

The optical linear polarisation map of M82 shows a centrosymmetric pattern of polarisation orientation, and a polarisation intensity that increases up to $\sim 30 \%$ at a distance of 200 arc seconds from the nucleus. 
A model of the galaxy has been constructed by assuming the body of the galaxy to be an edge-on luminous disk with a radial luminosity fall off given by $\exp (-\mathrm{kr})$ with $\mathrm{k}=2.0 \mathrm{kpc}^{-1}$. The disk is assumed to be situated in a large uniform dust cloud of dimensions $20 \mathrm{kpc}$. Further dust associated more closely with the galaxy is also assumed and this is taken to fall off as $\mathrm{k} \mathrm{Z}^{-\mathrm{n}}$ where $\mathrm{Z}$ is the height above the galactic $\mathrm{pl}$ ane and $\mathrm{k}$ and $\mathrm{n}$ are model parameters.

Using this model and assuming Rayleigh scattering by the surrounding dust of light originating in the galactic disk we have determined the Stokes parameters ( $Q, U, I)$ for various positions along the minor axis of the galaxy. We have compared the model predictions with the experimental data as shown in figure 1 and optimised the values of $k$ and $n$. We find a difference between the north and south directions in the galaxy and these are represented by the different values of the model parameters as indicated in figure 1. (This work was done in cooperation with H.G. Perkins, W.S. Pallister and R.G. Bingham.)
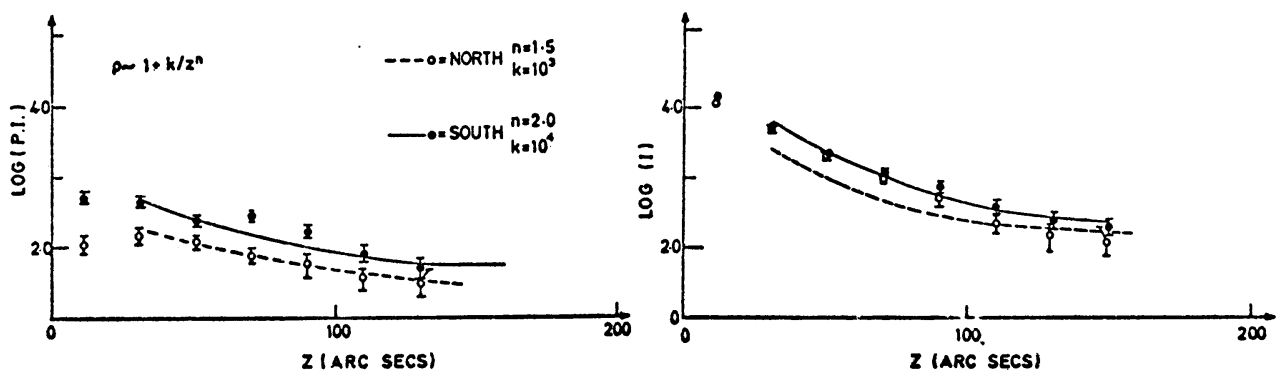

Fig. 1. Mode1 predictions to polarised intensity and total intensity for the galaxy M82.

VAN DER HULST: Does your model give a number for the density of the dust around M82?

SCARROTT: No. We normalise the predicted polarised intensity to our data at one point 100 arcseconds from the center of M82. To obtain an absolute number density of the dust we would need at least the luminosity of the nucleus and unseen face of M82; this is not known.

BALDWIN: Can you guess whether your data would fit models in which the dust, supposedly associated with M82 itself, is in fact at some other point in the line of sight?

SCARROTT: If the dust were between $M 82$ and ourselves the scattering that gives rise to the polarisations would be at small angles and consequently any polarisation would be very much less than the $20-30 \%$ observed. The dust therefore must be local to M82 to give a scattering geometry compatible with the observed polarisation.

GIOVANELLI: There is gas in the system of which M82 is a member and there are published synthesis maps of the gas distribution. How does 
it match the dust contribution that you assumed?

SCARROTT: We have yet to compare our dust density distribution with the gas distribution derivable from published radio maps. It has to be seen whether the radio maps are of sufficient detail to enable the comparison to be made; remember that our plots only extend to 150 arcseconds from the plane of the galaxy.

OORT: Isn't it difficult to imagine that such thin filaments as are seen in M82 could have persisted in the intergalactic medium?

COURTÈs: It is interesting to note that on the deep Ho photograph shown by Comte, the filaments exhibit a loop-like structure. This morphology should be in favour of a physical relation between M82 and the filaments and not of the intergalactic clouds interpretation.

SCARROTT: For our polarisation map an integration bin of 7 by 7 arcseconds was used and this smeared out any effects of the filaments in the presented polarisation pattern. Our model does not implicitly include the filaments and represents the general dust distribution around M82. Deep electronographs would best indicate the radial extent of the filaments. 